\title{
MODELO DE SIMULAÇÃO DA TEMPERATURA E UMIDADE RELATIVA DO AR NO INTERIOR DE ESTUFA PLÁSTICA
}

\author{
EDILSON COSTA ${ }^{1}$, PAULO A. M. LEAL ${ }^{2}$, RUY R. DO CARMO JÚNIOR ${ }^{3}$
}

RESUMO: A simulação dos parâmetros climáticos de temperatura e umidade relativa do ar no interior de uma estufa plástica, por meio do balanço de energia, pode propiciar ao produtor uma ferramenta de auxílio na tomada de decisão. Nesse propósito, realizou-se uma simulação das condições no interior de estufa plástica, em função de parâmetros externos e internos a ela. A simulação revelou uma temperatura no interior da estufa plástica de $23,6{ }^{\circ} \mathrm{C}$, e os sensores revelaram um valor médio de $24,1^{\circ} \mathrm{C}$ para o período de cultivo da alface. Para a umidade relativa no interior da estufa plástica, o valor simulado foi de $61,6 \%$, e o obtido com o auxílio de sensores foi de $66,0 \%$. Os valores simulados apresentaram-se próximos dos valores obtidos pelos sensores, mostrando que o modelo pode ser usado para a estimativa da temperatura e umidade relativa do ar no interior da estufa plástica.

PALAVRAS-CHAVE: estufa plástica, umidade relativa do ar, balanço de energia.

\section{SIMULATION MODEL OF AIR TEMPERATURE AND RELATIVE HUMIDITY IN TO PLASTIC GREENHOUSES}

SUMMARY: Simulation of climatic parameters inside air temperature and relative humidity of plastic greenhouse, trough energy balance, allows to growers a good technical tool on the decision making to improve the performance of inside environments. A simulation of internal conditions based on external and internal parameters was evaluated. The results showed the inside mean temperature of $23.6{ }^{\circ} \mathrm{C}$ in comparison with the experimental value of $24.1{ }^{\circ} \mathrm{C}$, for the cultivated period. The simulated relative humidity presented a value of $61.6 \%$ against $66.0 \%$ obtained by the sensors. The simulated values were closed to the values obtained by the sensors, which means that the model can be used to determine the internal conditions of plastic greenhouses.

KEYWORDS: plastic greenhouse, relative humidity of the air, energy balance.

\section{INTRODUÇÃO}

A simulação da umidade relativa e temperatura do ar no interior de estufa plástica pode propiciar ao produtor uma ferramenta de auxílio na tomada de decisão. Com os valores simulados pode-se determinar a necessidade ou não da utilização de tecnologia mais avançada para obter as condições necessárias ao desenvolvimento da cultura instalada, evitando custos elevados com energia, material e mão-de-obra, proporcionando ao agricultor melhor renda.

Essa simulação é feita em função do balanço de energia e consiste em determinar as condições climáticas do interior da estufa plástica quanto à temperatura e umidade relativa do ar. Esse balanço pode ser efetuado em qualquer instante do dia, mas, para o verão, é comumente realizado entre $12 \mathrm{e}$ $15 \mathrm{~h}$ e, para o inverno, entre $4 \mathrm{~h}$ e $30 \mathrm{~min}$ e $6 \mathrm{~h}$ e $30 \mathrm{~min}$, por serem horários críticos de temperaturas,

\footnotetext{
${ }^{1}$ Doutorando em Construções Rurais e Ambiência, FEAGRI/UNICAMP, Caixa Postal 6011, Campinas - SP, e-mail: mestrine@agr.unicamp.br

${ }^{2}$ Prof. Associado, FEAGRI/UNICAMP, Caixa Postal 6011, Campinas - SP, e-mail: pamleal@agr.unicamp.br

${ }^{3}$ Eng $^{\mathrm{o}} \mathrm{Agr}^{\mathrm{o}}$, Mestre em Engenharia Agrícola, FEAGRI/UNICAMP, Agroindustrial Lazzeri Ltda., e-mail: rrcarmo@yahoo.com.br

Recebido pelo Conselho Editorial em: 17-5-2000

Aprovado pelo Conselho Editorial em: 26-1-2004
} 
ou seja, ocorrência de máxima e mínima, respectivamente (ALBRIGHT, 1990). O mesmo cálculo também pode ser feito em função das médias dos parâmetros climáticos externos de um determinado período.

Este trabalho teve como objetivo testar um modelo de simulação da temperatura média do ar (Ti) e da umidade relativa média do ar (URi) num determinado período do ano, no interior de estufa plástica com laterais móveis, utilizando o balanço de energia, confrontando com os dados obtidos dos sensores.

\section{MATERIAL E MÉTODOS}

O experimento foi conduzido em um campo experimental da Faculdade de Engenharia Agrícola da Universidade Estadual de Campinas, utilizando estufa plástica de polietileno de baixa densidade (PEBD), modelo tipo capela e com laterais móveis, plástico transparente de espessura com 150 micras. Cultivou-se a alface (Lactuca sativa L.) variedade Vera, utilizando um sistema hidropônico com quatro bancadas triangulares de cultivo, no período de 17-11-1999 a 13-12-1999.

A simulação da temperatura média interna da estufa plástica (Ti), por meio do balanço de energia, utilizando apenas ventilação natural como renovação de ar, foi baseada nas equações de ASHRAE (1978) e HELLICKSON (1983), e envolveu as médias para o período de cálculo da temperatura do ar externo, umidade relativa do ar externo, radiação solar externa e velocidade do vento externa à estufa plástica, entre outras constantes físicas que podem ser extraídas de ALBRIGHT (1990), assim como dados do produto que podem ser extraídos de HONÓRIO \& BLISKA JÚNIOR (1996), ASHRAE (1978) e HELLICKSON (1983). Esse balanço de energia dentro de estufa plástica foi estimado pela eq.(1) (ASHRAE, 1978; HELLICKSON, 1983).

$$
\mathrm{Q}_{\mathrm{r}}+\mathrm{Q}_{\mathrm{m}}+\mathrm{Q}_{\mathrm{so}}+\mathrm{Q}_{\mathrm{sa}}+\mathrm{Q}_{\mathrm{ve}}=\mathrm{Q}_{\mathrm{ce}}+\mathrm{Q}_{\mathrm{sp}}+\mathrm{Q}_{\mathrm{sl}}+\mathrm{Q}_{\mathrm{vs}}+\mathrm{Q}_{\mathrm{ft}}+\mathrm{Q}_{\mathrm{tt}}
$$

em que,

$\mathrm{Q}_{\mathrm{r}}$ - calor sensível de respiração do produto, $\mathrm{W}$;

$\mathrm{Q}_{\mathrm{m}}$ - calor de motores, equipamentos, iluminárias, etc., W;

$\mathrm{Q}_{\text {so }}$ - calor sensível proveniente do Sol, W;

$\mathrm{Q}_{\mathrm{sa}}$ - calor sensível do sistema de aquecimento, $\mathrm{W}$;

$\mathrm{Q}_{\mathrm{ve}}$ - calor sensível do ar de ventilação de entrada (natural ou forçado), W;

$\mathrm{Q}_{\mathrm{ce}}$ - calor sensível de condução da estrutura, W;

$\mathrm{Q}_{\mathrm{sp}}$ - calor sensível transferido ao solo ou piso pelo perímetro, W;

$\mathrm{Q}_{\mathrm{sl}}$ - calor sensível convertido em calor latente dentro do espaço interno (evaporação da água dos vasos, sistemas de irrigação ou hidroponia e evapotranspiração), W;

$\mathrm{Q}_{\text {vs }}$ - calor sensível do ar de ventilação de saída (natural ou forçado), W;

$\mathrm{Q}_{\mathrm{ft}}$ - calor sensível usado para a fotossíntese, $\mathrm{W}$, e

$\mathrm{Q}_{\mathrm{tt}}$ - calor de transmitância térmica, $\mathrm{W}$.

Os valores dos termos $\mathrm{Q}_{\mathrm{r}}, \mathrm{Q}_{\mathrm{ft}}$ e $\mathrm{Q}_{\mathrm{sp}}$ são pequenos em relação aos valores dos demais termos e, muitas vezes, podem ser desconsiderados. Além disso, o valor de $\mathrm{Q}_{\mathrm{sl}}$ é muito difícil de ser medido, segundo ASHRAE (1978), podendo, também, ser desconsiderado.

Portanto, partindo da equação geral 1, eliminando os termos desprezíveis, obtém-se uma equação mais reduzida:

$\mathrm{Q}_{\mathrm{so}}+\mathrm{Q}_{\mathrm{ve}}=\mathrm{Q}_{\mathrm{ce}}+\mathrm{Q}_{\mathrm{sp}}+\mathrm{Q}_{\mathrm{vs}}+\mathrm{Q}_{\mathrm{tt}}$

O calor sensível proveniente do Sol (Qso), eq.(2), é definido como (ASHRAE, 1978; HELLICKSON, 1983): 


$$
\mathrm{Q}_{\mathrm{so}}=\tau \mathrm{I}_{\mathrm{e}} \mathrm{Ap}
$$

em que,

$\tau$ - transmitância da superfície da cobertura em relação à radiação solar global (ALBRIGHT, 1990), adimensional;

$\mathrm{I}_{\mathrm{e}}$ - radiação solar global externa, $\mathrm{W} \mathrm{m}^{-2}$, e

Ap - área do piso da estufa plástica, $\mathrm{m}^{2}$.

O calor sensível de respiração do produto $\left(\mathrm{Q}_{\mathrm{r}}\right)$, eq.(3), é definido como (ASHRAE, 1978; HELLICKSON, 1983):

$$
\mathrm{Q}_{\mathrm{r}}=0,10 \mathrm{Q}_{\mathrm{ft}}
$$

Outra forma:

$$
\mathrm{Q}_{\mathrm{r}}=\mathrm{mp} \mathrm{TR}
$$

em que,

$\mathrm{Q}_{\mathrm{ft}}$ - calor de fotossíntese, $\mathrm{W}$;

$\mathrm{mp}$ - massa do produto, $\mathrm{kg}$, e

TR - taxa de respiração do produto, $\mathrm{W} \mathrm{kg}^{-1}$.

O calor de fontes de energia mecânica, luminosas (iluminárias) e pessoas (Qm), eq.(4), é definido como (ASHRAE, 1978; HELLICKSON, 1983):

$$
\mathrm{Q}_{\mathrm{m}}=\mathrm{F} \mathrm{PE}+\text { iluminação }+ \text { pessoas }
$$

em que,

F - fator de potência do equipamento, adimensional;

$\mathrm{PE}$ - potência elétrica do equipamento, $\mathrm{W}$;

iluminação - calor perdido pela potência de lâmpadas, W, e pessoas - calor cedido por pessoas, $\mathrm{W}$.

O calor sensível do sistema de aquecimento (Qsa), eq.(5), é definido como (ASHRAE, 1978; HELLICKSON, 1983):

$$
\mathrm{Q}_{\mathrm{sa}}=\mathrm{PC}
$$

em que,

PC - potência calorífica da fonte aquecedora, W.

O calor sensível usado para a fotossíntese $\left(\mathrm{Q}_{\mathrm{ft}}\right)$, eq.(6), é definido como (ASHRAE, 1978; HELLICKSON, 1983):

$$
\mathrm{Q}_{\mathrm{ft}}=0,03 \mathrm{Q}_{\mathrm{so}}
$$

O calor sensível de condução da estrutura (Qce), eq.(7), é definido como (ASHRAE, 1978; HELLICKSON, 1983):

$$
\mathrm{Q}_{\mathrm{ce}}=\mathrm{U} \text { Ac } \Delta \mathrm{T}
$$

em que,

U - coeficiente global de transferência de calor do plástico, $\mathrm{W} \mathrm{m}^{-2} \mathrm{~K}^{-1}$ (ALBRIGHT, 1990);

Ac - área do contorno da estufa plástica com a lateral aberta ou fechada, $\mathrm{m}^{2}$;

$\Delta \mathrm{T}$ - temperatura interna - temperatura externa ( $\mathrm{Ti}$ - $\mathrm{Te}), \mathrm{K}, \mathrm{e}$

$\mathrm{Ti}$ - incógnita do balanço de energia, $\mathrm{K}$. 
O calor sensível transferido ao solo ou piso pelo perímetro $\left(\mathrm{Q}_{\mathrm{sp}}\right)$, eq.(8), é definido como (ASHRAE, 1978; HELLICKSON, 1983):

$$
\mathrm{Q}_{\mathrm{sp}}=\mathrm{F} \text { Per } \Delta \mathrm{T}
$$

em que,

$\mathrm{F}$ - fator perimetral, $\mathrm{W} \mathrm{m}^{-1} \mathrm{~K}^{-1}$;

Per - perímetro da estufa plástica, $\mathrm{m}$, e

$\Delta \mathrm{T}$ - temperatura interna - temperatura externa $(\mathrm{Ti}-\mathrm{Te}), \mathrm{K}$.

O calor de ventilação ( $Q_{v}$ ), eq.(9), é definido como (ASHRAE, 1978; HELLICKSON, 1983):

$$
\mathrm{Q}_{\mathrm{v}}=\mathrm{Q}_{\mathrm{ve}}-\mathrm{Q}_{\mathrm{vs}}=\overline{\mathrm{m}} \mathrm{cp} \Delta \mathrm{T}
$$

em que,

$\overline{\mathrm{m}}$ - fluxo mássico de ar seco, $\mathrm{kg} \mathrm{s}^{-1}, \mathrm{e}$

$\mathrm{cp}$ - calor específico do ar externo, $\mathrm{J} \mathrm{kg}^{-1} \mathrm{~K}^{-1}$.

A relação entre o fluxo volumétrico $(\overline{\mathrm{V}})$ e o fluxo mássico $\overline{\mathrm{m}}$, eq.(10), é descrita conforme a expressão (ASHRAE, 1978; HELLICKSON, 1983):

$$
\overline{\mathrm{V}}=\frac{\overline{\mathrm{m}}}{\rho \rho}
$$

em que,

$\overline{\mathrm{V}}$ - fluxo volumétrico, $\mathrm{m}^{3} \mathrm{~s}^{-1}, \mathrm{e}$

$\rho$ - densidade do ar externo, $\mathrm{kg} \mathrm{m}^{-3}$.

O fluxo volumétrico para a ventilação natural, eq.(11), é dado por (HELLICKSON, 1983):

$$
\overline{\mathrm{V}}=\mathrm{E} \mathrm{V}_{\mathrm{v}} \mathrm{A}_{\mathrm{a}}
$$

em que,

E- eficiência das aberturas, adimensional. (Para estruturas agrícolas adota-se $\mathrm{E}=0,35$ (HELLICKSON, 1983));

$\mathrm{V}_{\mathrm{v}}$ - velocidade média do vento externo a $2 \mathrm{~m}$ de altura , $\mathrm{m} \mathrm{s}^{-1}$, e

$\mathrm{A}_{\mathrm{a}}$ - área de abertura da estufa plástica, $\mathrm{m}^{2}$.

O calor sensível convertido em calor latente $\left(Q_{\mathrm{sl}}\right)$, eq.(12), é definido como (ASHRAE, 1978; HELLICKSON, 1983):

$$
\mathrm{Q}_{\mathrm{sl}}=\mathrm{ET} \text { Fp } \mathrm{Q}_{\mathrm{so}}
$$

em que,

ET - razão entre a radiação solar e a energia associada com a evapotranspiração da cultura, adimensional, recomenda-se 0,5 segundo (HELLICKSON, 1983), e

$\mathrm{Fp}$ - fator de cultivo (área de cultivo/área do piso), adimensional.

O calor de transmitância térmica (Qtt), eq.(13) é definido como (ASHRAE, 1978; HELLICKSON, 1983):

$$
\mathrm{Q}_{\mathrm{tt}}=\varepsilon_{\text {sup }} \gamma_{\mathrm{t}} \sigma \mathrm{Ap}\left(\mathrm{Ti}^{4}-\varepsilon_{\mathrm{ar}} \mathrm{Te}^{4}\right)
$$

em que, 
$\varepsilon_{\text {sup }}$ - emissividade do piso ou vegetação, adimensional (HELLICKSON, 1983);

$\gamma_{t}$ - transmitância térmica do plástico na reirradiação, adimensional (HELLICKSON, 1983);

Ap - área do piso, $\mathrm{m}^{2}$;

$\sigma$ - constante de Stefan Boltzmann, 5,678 $10^{-8} \mathrm{~W} \mathrm{~m}^{-2} \mathrm{~K}^{-4}$;

Te - temperatura externa, $\mathrm{K}$;

$\mathrm{Ti}$ - temperatura interna, $\mathrm{K}$, e

$\varepsilon_{\text {ar }}$ - emissividade do ar local, adimensional (HELLICKSON, 1983).

Para a determinação da umidade relativa interna média (URi), expressa em porcentagem, após a simulação da temperatura interna média (Ti), em K, utiliza-se o balanço de massa, definido pela eq.(14), o qual fornece a umidade absoluta interna média (Wi) (HELLICKSON, 1983).

$$
\overline{\mathrm{m}} \mathrm{Wi}=\overline{\mathrm{m}} \mathrm{We}+\mathrm{Map}
$$

em que,

$\overline{\mathrm{m}}$ - fluxo mássico de ar seco, $\mathrm{kg} \mathrm{s}^{-1}$;

Wi - umidade absoluta interna, $\mathrm{kg} \mathrm{kg}^{-1}$ (quilograma de vapor de $\mathrm{H}_{2} \mathrm{O}$ por quilograma de ar seco);

We - umidade absoluta externa, $\mathrm{kg} \mathrm{kg}^{-1}$ (quilograma de vapor de $\mathrm{H}_{2} \mathrm{O}$ por quilograma de ar seco);

Map - fluxo mássico de água produzida pelas plantas, $\mathrm{kg} \mathrm{s}^{-1}$.

Com Wi e Ti, utilizando o programa PLUS (Psychrometric Look-Up Substitute), escrito em linguagem Visual Basic e desenvolvido por ALBRIGHT (1990), determina-se a URi.

Para a medida da temperatura (Ti) e umidade relativa do ar (URi) internas, foi utilizado um psicrômetro constituído de termopares tipo $\mathrm{T}(\mathrm{Cu}-\mathrm{Co})$ e $\mathrm{J}$ (Fe-Co) com precisão de $\pm 0,5{ }^{\circ} \mathrm{C}$, calibrado pelo fabricante. A temperatura do ar externa (Te), a umidade relativa externa (URe) e a velocidade do vento externa (Vve) a $2 \mathrm{~m}$ de altura foram obtidas no posto meteorológico que dista $100 \mathrm{~m}$ do local do experimento. A radiação solar global externa (Ie) foi medida por piranômetro LICOR modelo $200 \mathrm{SZ}$.

Os dados de temperatura e umidade relativa do ar internas foram coletados por meio de um sistema automatizado. Os dados foram medidos a cada 10 segundos e armazenados, sendo a média desses medidos a cada 30 minutos para a caracterização da temperatura horária e diária e obtenção da média no período, objetivo da comparação. A temperatura obtida do posto meteorológico foi armazenada a cada 10 minutos. A radiação solar global externa foi coletada num sistema de aquisição de dados LICOR modelo Li-1400, que armazenava os dados diariamente de hora em hora, entre 7 e $17 \mathrm{~h}$.

A seguir, são apresentados os dados de entrada no modelo na eq.(1).

TABELA 1. Dados de entrada do modelo de simulação.

\begin{tabular}{llll}
\hline $\mathrm{Q}_{\mathrm{r}}=\mathrm{Qft} 0,1$ & $\mathrm{~m}=\mathrm{V} \rho$ & $\mathrm{Ti}=$ incógnita, em $\mathrm{K}$ & $\mathrm{Q}_{\mathrm{ft}}=\mathrm{Qso} 0,03$ \\
$\mathrm{Q}_{\mathrm{m}}=0$ & $\mathrm{~V}=\mathrm{E} \mathrm{Vv} \mathrm{Aa}$ & $\mathrm{Q}_{\mathrm{ce}}=\mathrm{U}$ Ac $\Delta \mathrm{T}$ & $\mathrm{Q}_{\mathrm{tt}}=\varepsilon_{\text {sup }} \gamma_{\mathrm{t}} \sigma \mathrm{Ap}\left(\mathrm{Ti}^{4}-\varepsilon_{\mathrm{ar}} \mathrm{Te}^{4}\right)$ \\
$\mathrm{Q}_{\mathrm{so}}=\tau \mathrm{I}$ Ap & $\mathrm{E}=0,35$ & $\mathrm{U}=7,14$ & $\varepsilon_{\mathrm{sup}}=0,85$ \\
$\tau=0,95$ & $\mathrm{Vv}=1,53$ & $\mathrm{Ac}=139,77$ & $\gamma_{\mathrm{t}}=0,8$ \\
$\mathrm{I}=569,84$ & $\mathrm{Aa}=19,06$ & $\mathrm{Q}_{\mathrm{sp}}=\mathrm{F}$ Per $\Delta \mathrm{T}$ & $\sigma=5,6710^{-8}$ \\
$\mathrm{Ap}=74,75$ & $\rho=1,09$ & $\mathrm{~F}=1,15$ & $\mathrm{Ap}=74,75$ \\
$\mathrm{Q}_{\mathrm{sa}}=0$ & $\mathrm{cp}=1006$ & Per $=36$ & $\varepsilon_{\mathrm{ar}}=0,8$ \\
$\mathrm{Q}_{\mathrm{v}}=\mathrm{m} \mathrm{Cp} \Delta \mathrm{T}$ & $\Delta \mathrm{T}=(\mathrm{Ti}-\mathrm{Te})$ & $\mathrm{Q}_{\mathrm{sl}}=0$ & \\
\hline
\end{tabular}


Resolvendo $\mathrm{Q}_{\mathrm{so}}+\mathrm{Q}_{\mathrm{r}}+\mathrm{Q}_{\mathrm{m}}+\mathrm{Q}_{\mathrm{sa}}-\mathrm{Q}_{\mathrm{v}}-\mathrm{Q}_{\mathrm{ce}}-\mathrm{Q}_{\mathrm{ft}}-\mathrm{Q}_{\mathrm{sp}}-\mathrm{Q}_{\mathrm{sl}}-\mathrm{Q}_{\mathrm{tt}}=0$, obtém-se Ti.

Para a determinação da URi [eq.(14)], assumiu-se uma evapotranspiração para a cultura da alface de 3,29 $\mathrm{mm} \mathrm{dia}^{-1}\left(3,29 \mathrm{~L} \mathrm{~m}^{-2}\right.$ - (GONÇALVES et al. 2001)) e, tendo uma área de cultivo de $56,4 \mathrm{~m}^{2}$, determinou-se o fluxo de produção de água pelas plantas (Map).

Map = fluxo de massa de água produzida pelas plantas $=$ evapotranspiração $\mathrm{x}$ área de cultivo $=$ Map $=\frac{3,29 \mathrm{~L}}{\mathrm{~m}^{2} \text { dia }} \frac{56,4 \mathrm{~m}^{2}}{1} \frac{1 \text { dia }}{86400 \mathrm{~s}} \frac{1 \mathrm{~kg} \text { água }}{1 \mathrm{~L}}=0,00214764 \mathrm{kgv} \mathrm{s}^{-1}(\mathrm{kgv}=$ quilograma de vapor

d'água contida no ar por segundo). Utiliza-se vazão mássica, pois a vazão volumétrica é menos precisa.

O fluxo mássico de ar $(\overline{\mathrm{m}})$ que entra na estufa foi determinado multiplicando-se o fluxo $\operatorname{volumétrico~}\left(\overline{\mathrm{V}}=\mathrm{E} \mathrm{V}_{\mathrm{v}} \mathrm{A}_{\mathrm{a}}\right)$ pela densidade do $\operatorname{ar}(\rho)$.

E - eficiência das aberturas $=0,35$;

$\mathrm{Vv}$ - velocidade do vento a $2 \mathrm{~m}=1,53 \mathrm{~m} \mathrm{~s}^{-1}$;

Aa - área de abertura $=19,06 \mathrm{~m}^{2}, \mathrm{e}$

$\overline{\mathrm{m}}=\mathrm{E} \mathrm{A}_{\mathrm{a}} \mathrm{V}_{\mathrm{v}} \rho=0,3519,061,531,09=11,12 \mathrm{~kg} \mathrm{~s}^{-1}$.

A umidade absoluta externa We é obtida com auxílio do "software PLUS" (ALBRIGHT, 1990). Entra-se com o valor da temperatura externa $\left(20,8{ }^{\circ} \mathrm{C}\right)$ e com o valor da umidade relativa externa $(72,2 \%)$ e obtém-se We.

$\mathrm{We}=$ umidade absoluta externa $=0,0120327 \mathrm{~kg} \mathrm{~kg}^{-1}$

Com esses dados, utilizando a eq.(14),

$\overline{\mathrm{m}} \mathrm{Wi}=\overline{\mathrm{m}} \mathrm{We}+\mathrm{Map}$

$\overline{\mathrm{m}}=11,12 \mathrm{~kg} \mathrm{~s}^{-1}$;

Map $=0,00214764 \mathrm{~kg} \mathrm{~s}^{-1}$, e

$\mathrm{We}=0,0120327 \mathrm{~kg} \mathrm{kga}^{-1}$.

Determinou-se a umidade absoluta interna (Wi), por meio da resolução da eq.(14).

$$
\begin{aligned}
\mathrm{Wi} & =\frac{(\overline{\mathrm{m}} \mathrm{We}+\mathrm{Map})}{\overline{\mathrm{m}}} \\
\mathrm{Wi} & =0,012052 \mathrm{~kg} \mathrm{~kg}^{-1}
\end{aligned}
$$

\section{RESULTADOS E DISCUSSÃO}

Utilizando-se de um pacote de computação científica, obteve-se a temperatura média interna em Kelvin $(\mathrm{K})$, resolvendo o balanço de energia $\mathrm{Ti}=296,6 \mathrm{~K}=23,6{ }^{\circ} \mathrm{C}$.

Com a umidade absoluta interna $\mathrm{Wi}=0,012052 \mathrm{~kg} \mathrm{~kg}^{-1}$ e a temperatura interna de $\mathrm{Ti}=23,6{ }^{\circ} \mathrm{C}$, determinou-se a umidade relativa interna (URi $=61,6 \%$ ), com o auxílio do software PLUS (ALBRIGHT, 1990).

$\mathrm{Na}$ Tabela 2, expressam-se os valores médios da temperatura interna $\left(\mathrm{Ti}-{ }^{\circ} \mathrm{C}\right)$, temperatura externa $\left(\mathrm{Te}-{ }^{\circ} \mathrm{C}\right)$, umidade relativa interna (URi - \%), umidade relativa externa (Ure - \%), radiação 
global externa $\left(\mathrm{Ie}-\mathrm{Wm}^{-2}\right.$ ) e velocidade do vento externa a $2 \mathrm{~m}$ de altura (Vve $-\mathrm{m} \mathrm{s}^{-1}$ ), temperatura interna do ar simulada (Tis $-{ }^{\circ} \mathrm{C}$ ) e umidade relativa interna simulada $\left(\mathrm{Ti}-{ }^{\circ} \mathrm{C}\right.$ ) obtidos durante $\mathrm{o}$ experimento.

TABELA 2. Dados médios diários obtidos e simulados no período estudado.

\begin{tabular}{ccclccccc}
\hline Data & Ie & Vve & URe & URi & URis & Te & Ti & Tis \\
\hline 17-nov. & 727,01 & 2,47 & 62,8 & 52,4 & 54,2 & 18,16 & 21,3 & 20,5 \\
18-nov. & 782,34 & 2,09 & 65,5 & 54,2 & 54,5 & 18,94 & 22,6 & 21,9 \\
19-nov. & 623,88 & 1,88 & 69 & 59,2 & 58,9 & 19,73 & 23,3 & 22,3 \\
20-nov. & 354,07 & 1,24 & 76,8 & 62,7 & 68,1 & 19,28 & 22,5 & 21,2 \\
21-nov. & 496,51 & 0,88 & 74,6 & 62,4 & 58,8 & 19,95 & 23,2 & 23,8 \\
22-nov. & 679,05 & 1,24 & 71,4 & 59,1 & 55,7 & 20,43 & 24,7 & 24,5 \\
23-nov. & 763,33 & 1,36 & 64,8 & 54,6 & 49,9 & 20,60 & 24,6 & 24,9 \\
24-nov. & 840,95 & 1,56 & 58 & 47,5 & 45,0 & 22,09 & 26,5 & 26,3 \\
25-nov. & 762,02 & 1,39 & 53,1 & 44,6 & 41,3 & 23,28 & 27,9 & 27,5 \\
26-nov. & 292,94 & 0,95 & 80,1 & 81,9 & 71,1 & 19,93 & 22,3 & 21,9 \\
27-nov. & 759,16 & 1,24 & 65,5 & 54,8 & 49,6 & 22,49 & 26,6 & 27,2 \\
28-nov. & 739,54 & 2,39 & 53,9 & 51,7 & 46,3 & 21,73 & 25,6 & 24,2 \\
29-nov. & 816,29 & 2,22 & 65,3 & 58,7 & 54,5 & 20,91 & 24,4 & 23,9 \\
30-nov. & 616,28 & 2,58 & 69,4 & 62,1 & 61,8 & 20,84 & 24,2 & 22,7 \\
1-dez. & 843,25 & 2,17 & 65,6 & 59,9 & 54,2 & 21,71 & 25,0 & 24,9 \\
2-dez. & 722,44 & 1,45 & 64,5 & 58,3 & 51,3 & 22,46 & 25,6 & 26,3 \\
3-dez. & 575,57 & 1,25 & 72,2 & 65,4 & 58,8 & 21,84 & 25,6 & 25,3 \\
4-dez. & 236,38 & 1,14 & 89,4 & 87 & 82,7 & 19,48 & 22,0 & 20,7 \\
5-dez. & 440,72 & 1,05 & 69,5 & 67,9 & 58,3 & 22,50 & 25,8 & 25,4 \\
6-dez. & 513,95 & 1,17 & 76,2 & 72,4 & 62,9 & 20,98 & 24,2 & 24,1 \\
7-dez. & 571,84 & 1,33 & 79,1 & 72,8 & 65,1 & 21,33 & 24,8 & 24,5 \\
8-dez. & 637,73 & 1,22 & 80,8 & 77,7 & 63,9 & 21,66 & 25,5 & 25,6 \\
9-dez. & 621,79 & 1,81 & 78,6 & 73,5 & 66,9 & 22,02 & 25,3 & 24,7 \\
10-dez. & 398,01 & 1,52 & 83,1 & 77,2 & 74,1 & 20,65 & 22,8 & 22,5 \\
11-dez. & 226,05 & 1,19 & 86,7 & 86,9 & 80,8 & 19,35 & 21,0 & 20,5 \\
12-dez. & 183,00 & 1,38 & 81,3 & 84,6 & 77,7 & 19,72 & 21,9 & 20,5 \\
13-dez. & 161,54 & 1,21 & 91,6 & 93,3 & 96,0 & 18,67 & 20,8 & 19,4 \\
\hline Média & 569,84 & 1,53 & 72,2 & 66,0 & 61,6 & 20,8 & 24,1 & 23,6 \\
\hline
\end{tabular}

Na Figura 1, apresenta-se a variação das temperaturas interna e externa do ar à estufa plástica. Observa-se que a temperatura interna média foi maior que a temperatura externa média durante o período de experimento, devido ao acúmulo de energia no interior da estufa plástica em função das características do material plástico, conforme observações encontradas em CAMACHO et al. (1995), FARIAS et al. (1993) e CUNHA \& ESCOBEDO (2003).

$\mathrm{Na}$ Figura 2, apresenta-se a variação das umidades relativas média diária interna e externa. Observa-se que a umidade relativa média interna foi menor que a umidade relativa externa média durante o período do experimento, mesmo as plantas produzindo vapor d'água no interior da estufa plástica. A umidade relativa do ar apresenta-se inversamente proporcional à temperatura do ar, pois à medida que a temperatura do ar aumenta em função do aumento da disponibilidade energética na superfície do solo (radiação solar global), a umidade relativa do ar diminui em função do aquecimento do ar. Considerando que a estufa plástica apresenta um menor volume de ar em relação à condição externa, ocorre, então, a inibição do processo convectivo devido à barreira imposta pela presença do filme plástico usado na estufa (CUNHA \& ESCOBEDO, 2003). 


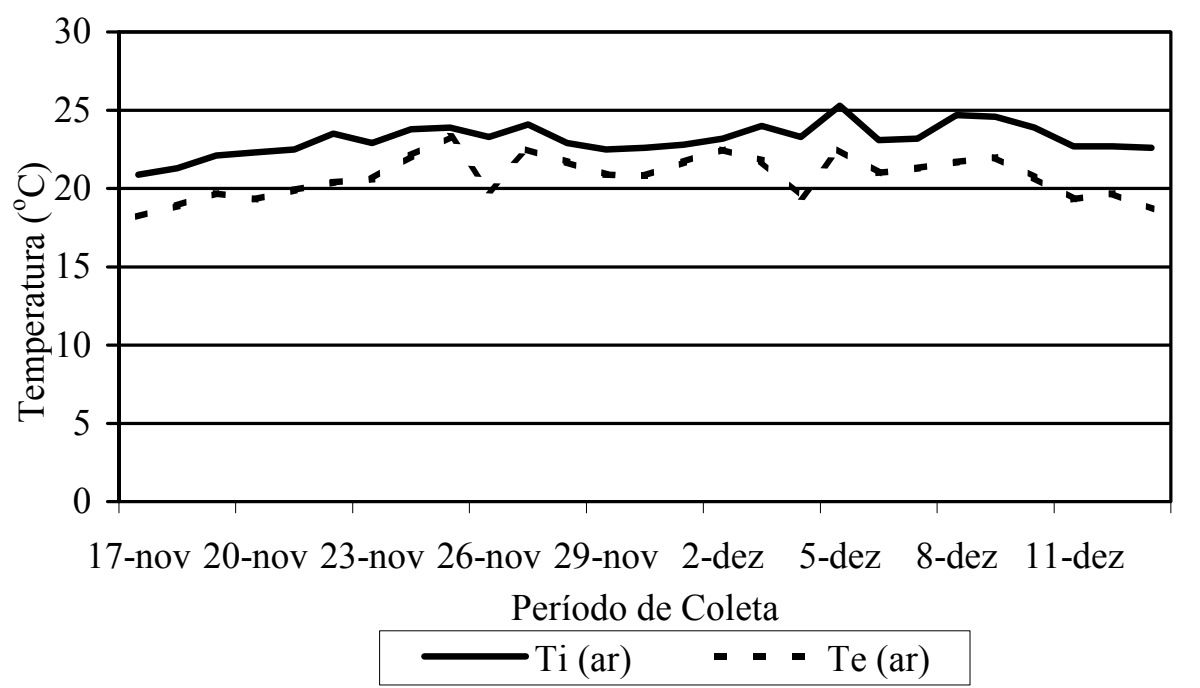

FIGURA 1. Variação da temperatura no interior da estufa plástica (Ti) e temperatura externa à estufa plástica (Te), ao longo do período estudado.

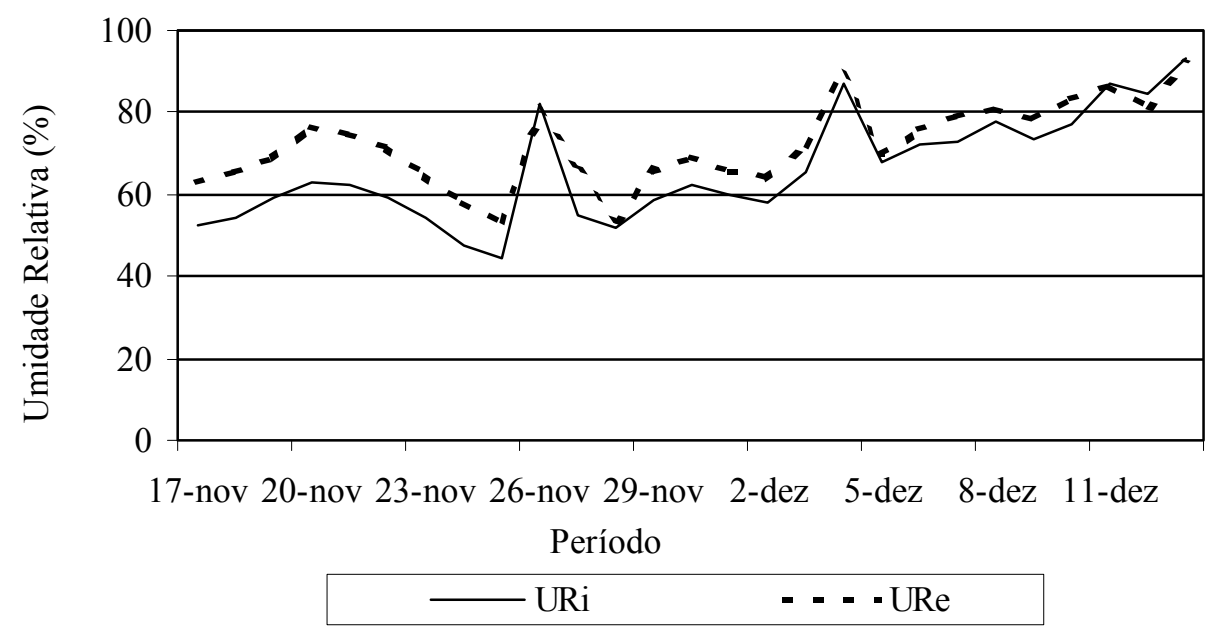

FIGURA 2. Variação da umidade relativa no interior da estufa plástica (URi) e umidade relativa externa à estufa plástica (URe), ao longo do período estudado.

Na Figura 3, apresenta-se a variação da temperatura no interior da estufa plástica (temperatura medida e temperatura simulada).

Analisando a Figura 3, observa-se que foram similares as temperaturas do ar medidas pelos sensores e as temperaturas do ar simuladas. Observando a temperatura média interna medida durante $o$ período do experimento $\left(24,1^{\circ} \mathrm{C}\right)$ e comparando ao valor da temperatura média interna simulada $\left(23,6{ }^{\circ} \mathrm{C}\right)$, Tabela 2 , observa-se que a temperatura simulada é $0,5^{\circ} \mathrm{C}$ menor que a temperatura medida, o que valida o modelo, pois a análise estatística a 5\% de significância não apresentou diferença. Essa diferença pode ser considerada pequena, uma vez que os sensores de temperatura possuem uma precisão de $\pm 0,5^{\circ} \mathrm{C}$. Dessa forma, consegue-se apresentar um valor modelado muito próximo do real, permitindo aos produtores, que não possuem condições de adquirir equipamentos e aparelhos, fazer uma análise do ambiente interno de produção antes mesmo de estarem produzindo e, portanto, optarem pela aquisição de sensores ou equipamentos com grau tecnológico de manejo que mais lhes convier. 


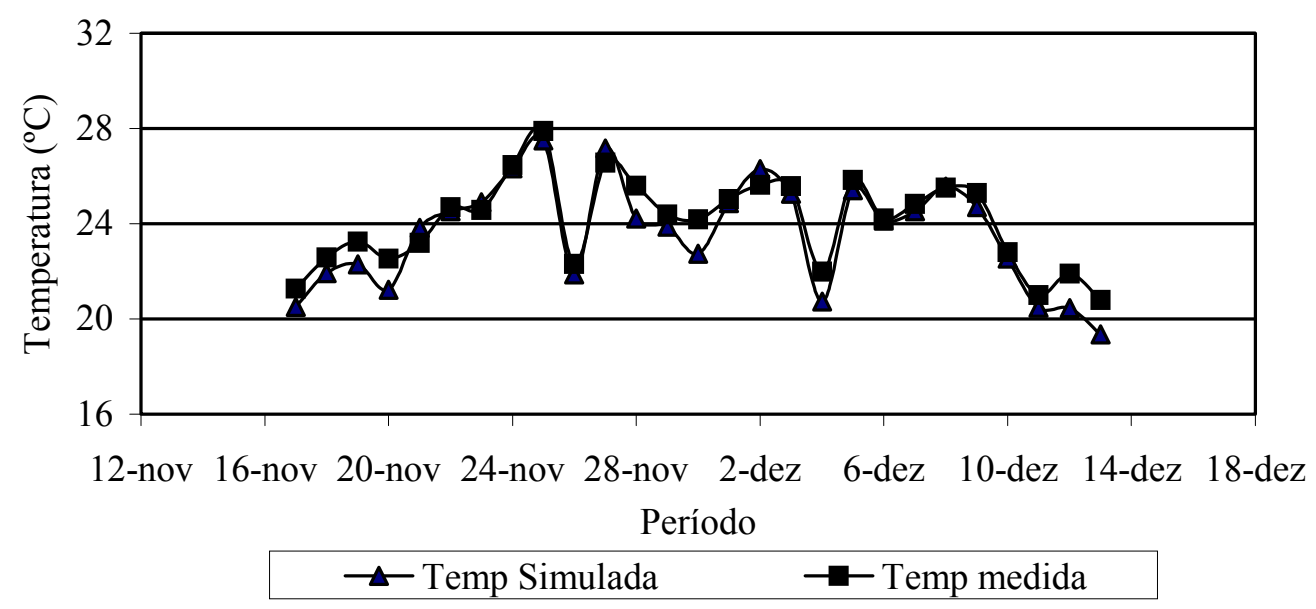

FIGURA 3. Variação da temperatura do ar ao longo do período estudado.

Na Figura 4, apresenta-se a variação da umidade relativa simulada (URis) e da umidade relativa medida pelos sensores durante o experimento. Analisando a Figura 4, observa-se que foram similares as umidades relativas medidas pelos sensores e as umidades relativas simuladas.

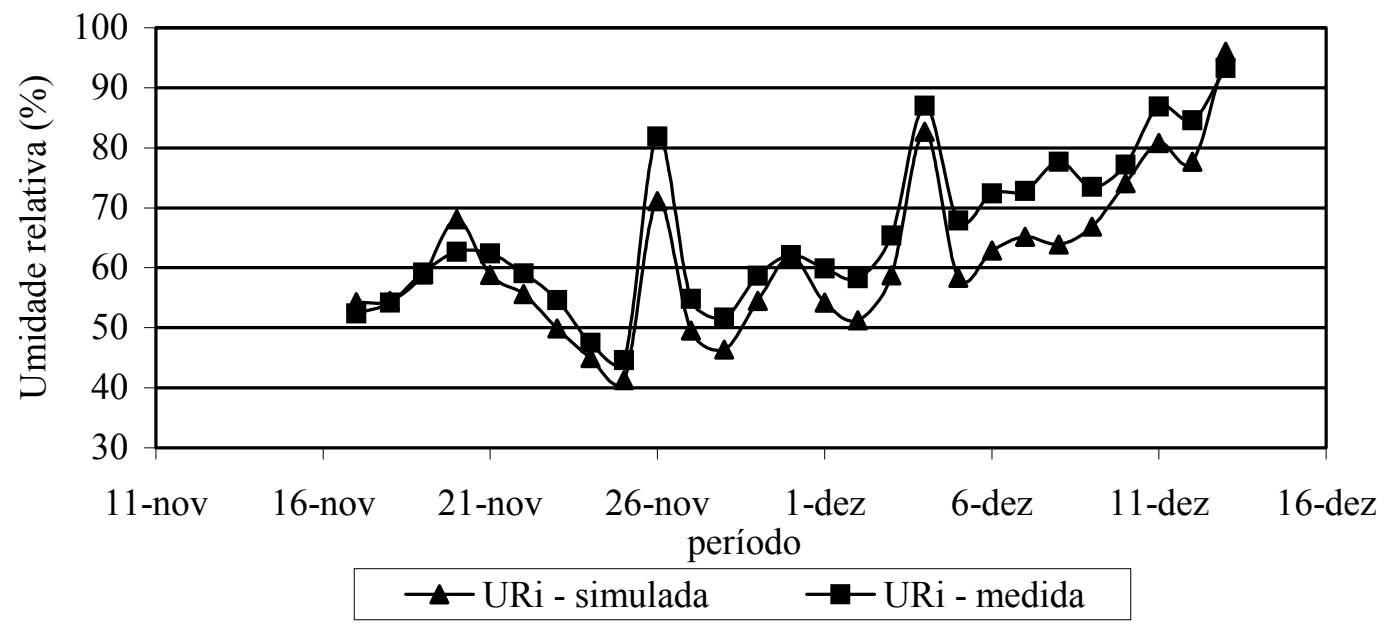

FIGURA 4. Variação da umidade relativa do ar ao longo do período.

Comparando o valor médio URis $=61,6 \%$ com o calculado com o auxílio dos sensores de temperaturas de bulbo seco e úmido do ar, 66,0\%, conforme Tabela 2, tem-se, por meio da simulação, uma boa aproximação também para a umidade relativa do ar, uma vez que a análise estatística não apresentou diferença a 5\% de significância (Tabela 4).

Fazendo-se uma análise estatística de comparação de médias, considerando os valores médios diários como repetição, obtém-se Tabelas 3 e 4 ("software ESTAT").

Analisando as Tabelas 3 e 4, observa-se que, tanto o teste $\mathrm{F}$ quanto o teste de Tukey, não apresentaram diferenças estatísticas entre os valores simulados e os valores medidos pelos sensores, para as temperaturas de bulbo seco e bulbo úmido do ar, calculando a umidade relativa do ar, no período analisado. Isso valida o modelo de simulação na utilização da estimativa da temperatura e umidade relativa do ar para o interior da estufa plástica. 
TABELA 3. Análise de variância do experimento (temperatura do ar) e teste de Tukey - "software ESTAT".

\begin{tabular}{|c|c|c|c|c|c|c|}
\hline C. Variação & G.L. & S.Q. & Q.M. & $\mathrm{F}$ & \multicolumn{2}{|c|}{ Teste de Tukey } \\
\hline Tratamentos & 1 & 3,0341 & 3,0341 & $0,72 \mathrm{NS}$ & DMS (Tukey) $=$ & $=1,1245$ \\
\hline Resíduo & 52 & 220,2319 & 4,2352 & & Trat. & Média \\
\hline Total & 53 & 223,2659 & & & & 7 \\
\hline $\mathrm{DP}=2,0580$ & & $\mathrm{EPM}=$ & 0,3961 & & 24 & $4,0741 \mathrm{~A}$ \\
\hline $\bar{\mu}=23,8370$ & & $\mathrm{CV}=$ & 8,63 & & 23 & $3,6000 \mathrm{~A}$ \\
\hline
\end{tabular}

TABELA 4. Análise de variância do experimento (umidade relativa do ar) e teste de Tukey "software ESTAT".

\begin{tabular}{|c|c|c|c|c|c|c|}
\hline C. Variação & G.L. & S.Q. & Q.M. & $\mathrm{F}$ & \multicolumn{2}{|c|}{ Teste de Tukey } \\
\hline Tratamentos & 1 & 268,4474 & 268,4474 & $1,61 \mathrm{NS}$ & DMS $($ Tukey $)=$ & 1,1245 \\
\hline Resíduo & 52 & 8678,8326 & 166,9006 & & Trat. & Média \\
\hline Total & 53 & 8947,2800 & & & & \\
\hline 12,9190 & & $\mathrm{EPM}=$ & 4863 & & 2 & $6,0296 \mathrm{~A}$ \\
\hline 63,8000 & & $\mathrm{CV}=2$ & & & 1 & $1,5704 \mathrm{~A}$ \\
\hline
\end{tabular}

Salienta-se que se pode fazer uma análise mais criteriosa do modelo de simulação utilizando dados horários e/ou diários para períodos mais longos, reais e simulados, fazendo uma comparação de médias entre os valores obtidos no real e no simulado. Dessa forma, pode-se obter uma análise mais criteriosa na correlação entre a simulação e o real. No entanto, CUNHA \& ESCOBEDO (2003) observaram pequenas diferenças nos valores médios diários e médios ao longo do período analisado, com relação à temperatura e umidade relativa do ar entre a estufa plástica e condições de campo, com valores médios ao longo do período de temperatura do ar de $19,41{ }^{\circ} \mathrm{C}$ e $18,74{ }^{\circ} \mathrm{C}$, e de umidade relativa do ar de $67,14 \%$ e $67,92 \%$, respectivamente, para a estufa plástica e a campo; valores semelhantes aos encontrados por FARIAS et al. (1993).

\section{CONCLUSÕES}

Para o período analisado, os resultados obtidos foram adequados para simular a temperatura e a umidade relativa do ar no interior da estufa plástica, em função de não haver diferença estatística, a 5\% de significância, entre o resultado do modelo e o valor medido.

No entanto, ressalta-se que se podem fazer comparações, entre valores simulados e valores medidos, em períodos maiores, utilizando dados horários e/ou diários, e com isso obter maior precisão do modelo.

\section{AGRADECIMENTOS}

À FAPESP (Processos n os 98/10476-0, 98/10477-7, 98/10478-3, 99/02249-7, 99/02676-2) e ao CNPq (131.919/1998-9).

\section{REFERÊNCIAS BIBLIOGRÁFICAS}

ALBRIGHT, L.D. Environment control for animals and plants. St. Joseph: ASAE, 1990. 453 p.

ASHRAE. Environment control for animals and plants. In: ASHRAE Handbook of Applications. USA: ASHRAE, 1978. Chapter 22, p.22.1-22.20. 
BOILY, R. Estufa - controle do meio ambiente. Campinas: FEAGRI/UNICAMP, 1999. 75 p. (Apostila)

CAMACHO, J.M.; ASSIS, F.N.; Martins, S.R.; MENDEZ, M.E.G. Avaliação de elementos meteorológicos em estufa plástica em Pelotas. Revista Brasileira de Agrometeorologia, Santa Maria, v.3, n.1, p.19-24, 1995.

CUNHA, A.R.; ESCOBEDO, J.F. Alterações micrometeorológicas causadas pela estufa plástica e seus efeitos no crescimento e produção da cultura de pimentão. Revista Brasileira de Agrometeorologia, Santa Maria, v.11, n.1, p.15-27, 2003.

FARIAS, J.R.B.; BERGAMASCHI, H.; MARTINS, S.R.; BERLATO, M.A.; OLIVEIRA, A.C.B. Alterações na temperatura e umidade relativa do ar provocadas pelo uso de estufa plástica. Revista Brasileira de Agrometeorologia, Santa Maria, v.1, n.1, p.51-62, 1993.

GONÇALVES, A.O.; OLIVEIRA, J.C.; PERES, J.G. Determinação das necessidades de água da cultura da alface (Lactuca sativa L.) sob condições protegidas. In. CONGRESSO DE INICIAÇÃO CIENTIFICA, 6., 2001, São Carlos. Anais... São Carlos: UFSCAR/PRG, 2001. 3 p.

HELLICKSON, M.A. Ventilation of agricultural structures. St. Joseph: ASAE, 1983. 372 p.

HONÓRIO, S.L.; BLISKA JÚNIOR, A. Cartilha tecnológica: plasticultura e estufa. Campinas: FEAGRI/UNICAMP, 1996. 51 p. 